\title{
MtrA regulation of essential peptidoglycan cleavage in Mycobacterium tuberculosis during infection
}

2 Eliza J. R. Peterson ${ }^{1, *}$, Aaron N Brooks ${ }^{1, \uparrow}$, David J. Reiss ${ }^{1, *}$, Amardeep Kaur ${ }^{1}$, Wei-Ju Wu ${ }^{1}$, Robert

3 Morrison $^{2}$, Vivek Srinivas ${ }^{1}$, Serdar Turkarslan ${ }^{1}$, Min Pan $^{1}$, Warren Carter ${ }^{1}$, Mario L. Arrieta-Ortiz ${ }^{1}$, Rene

4 A. Ruiz ${ }^{1}$, Apoorva Bhatt ${ }^{3}$, Nitin S. Baliga ${ }^{1,4,5,6, *}$

5

${ }^{1}$ Institute for Systems Biology, Seattle, WA, USA

${ }^{2}$ Laboratory of Malaria, Immunology and Vaccinology, National Institute of Allergy and Infectious

8 Diseases, NIH, Bethesda, Maryland, USA

$9{ }^{3}$ School of Biosciences and Institute of Microbiology and Infection, University of Birmingham,

10 Birmingham, UK

$11{ }^{4}$ Departments of Biology and Microbiology, University of Washington, Seattle, WA, USA

$12{ }^{5}$ Molecular and Cellular Biology Program, University of Washington, Seattle, WA, USA

$13 \quad{ }^{6}$ Lawrence Berkeley National Lab, Berkeley, CA, USA

$14 \dagger$ Current affiliation: Inscripta, Inc. Boulder, CO, USA

$15 *$ Corresponding author

16 Eliza J. R. Peterson: eliza.peterson@isbscience.org

17 Nitin S. Baliga: nitin.baliga@isbscience.org (lead contact)

Abstract: The success of Mycobacterium tuberculosis (Mtb) is largely due to its ability to withstand multiple stresses encountered in the host. Here, we present a data-driven model that captures the dynamic interplay of environmental cues and genome-encoded regulatory programs in Mtb. The model captures the genome-wide distribution of cis-acting gene regulatory elements and the conditional influences of transcription factors at those elements to elicit environment-specific responses. Analysis of transcriptional responses that may be essential for Mtb to survive acidic stress within the maturing macrophage, identified regulatory control by the MtrAB two-component signal system. Using genome-wide transcriptomics as well as imaging studies, we have characterized the MtrAB circuit by tunable CRISPRi knockdown in both Mtb and the non-pathogenic organism, M. smegmatis (Msm). These experiments validated the essentiality of MtrA in Mtb, but not Msm. We identified that MtrA regulates multiple enzymes that cleave cell wall peptidoglycan and is required for efficient cell division. Moreover, our results suggest that peptidoglycan cleavage, regulated by MtrA, is necessary for Mtb to survive intracellular stress. Further, we present MtrA as an attractive drug target, as even weak repression of $m t r A$ results in loss of Mtb viability and completely clears the bacteria with low-dose isoniazid or rifampicin treatment. 
Mycobacterium tuberculosis (Mtb) infection occurs by inhalation of bacilli-containing aerosols. Mtb initially colonize the lungs, where alveolar macrophages phagocytize the bacteria and sequester them in a phagosome. As Mtb navigate their intraphagosomal environment, they rely on gene regulatory networks (GRNs) that have evolved to evade the bactericidal properties of the maturing phagosome (e.g., acidic pH, reactive oxygen and nitrogen species, nutrient deprivation). Building accurate and comprehensive GRN models can inform how Mtb survives the myriad of stresses within the host environment. A fully characterized GRN of Mtb would also enable actionable hypotheses for identifying novel therapeutic strategies that could disrupt Mtb's successful tolerance to stress and better control infection.

The GRN structure is encoded in an organism's genome as transcription factor (TF) binding sites, referred to as gene regulatory elements (GREs). GREs, 6-20 nucleotide DNA sequences, are invariant to environmental stresses. However, environmental and genetic perturbations alter the affinity of TFs to bind GREs, which in turn modulate the transcriptome in a condition-specific manner. Therefore, the goal of reconstructing a GRN is to produce an unbiased genome-wide map of GREs, including information about what regulators bind to those sequences, in what contexts they are bound, and, importantly, how TF-binding throughout the genome ultimately influences cellular physiology.

We previously reported models of Escherichia coli and Halobacterium salinarum ${ }^{1}$ that realized these goals of GRN inference. The models were constructed with EGRIN 2.0, which provided a number of advancements to the previous Environment and Gene Regulatory Influence Networks (EGRIN)2 ${ }^{2}$ EGRIN models, learned by cMonkey $^{3,4}$ and Inferelator ${ }^{5}$, have accurately predicted conditional regulatory interactions in a number of organisms including $\mathrm{Mtb}^{6-13}$; yet, these network models had some limitations in probabilistically uncovering conditional co-regulation of genes by specific TFs and GREs, especially in under-represented environmental conditions in the transcriptome compendium. Therefore, we developed EGRIN 2.0 to overcome these shortcomings and model the organization of GREs within every promoter and link the contexts in which they act to the conditional co-regulation of genes. Importantly, the improvements and algorithmic components of EGRIN 2.0 have been rigorously tested and validated in prior studies $^{1,3-5}$; readers are encouraged to refer to the original papers for more detail. As such, this study was able to focus on revealing regulatory behaviors that are critical to Mtb pathogenesis, with experimental validation of model-based predictions.

One of the earliest environmental cues encountered by Mtb during infection is a drop in $\mathrm{pH}$ during maturation of the macrophage phagosome. In fact, $\mathrm{Mtb}$ responds transcriptionally to the acidic $\mathrm{pH}$ of the macrophage within 20 minutes of phagocytosis ${ }^{14}$, manifesting in a number of physiological responses, 
is accompanied by complex changes (e.g., cell wall structure, metabolic activity, target abundance) that can affect the pathogen's susceptibility to antibiotics.

Here, we have uncovered the regulatory programs that control Mtb's transcriptional response to diverse host-relevant environmental cues, to understand what makes Mtb such a successful and difficult to treat pathogen. We constructed an EGRIN 2.0 model for Mtb and used the mechanistic detail captured to discover regulatory mechanisms responsible for the pathogen's $\mathrm{pH}$-adapted lifestyle. Specifically, using EGRIN 2.0 we formulated the hypotheses that the response regulator, MtrA conditionally modulates cell division during acidic stress by regulating multiple cell wall peptidoglycan (PG) cleavage enzymes. Using tunable CRISPRi gene silencing to knockdown mtrA, we have validated the EGRIN 2.0-predicted regulatory activity of MtrA, and demonstrate that even minimal mtrA knockdown is lethal to Mtb and results in complete clearance with treatment of frontline antibiotics. Together, these results describe the extreme vulnerability of the MtrA regulon and its potential as a novel therapeutic target.

\section{RESULTS}

\section{Constructing EGRIN 2.0 for Mtb}

EGRIN 2.0 is an ensemble framework that aggregates associations across genes, GREs, and environments from many individual EGRIN models, each trained on a subset of gene expression data. The aggregated, post-processed ensemble of EGRIN models is referred to as EGRIN 2.0, which details the organization of GREs within every promoter and identifies conditionally co-regulated gene modules, known as corems. The Mtb EGRIN 2.0 model was constructed using the same workflow as the previous EGIRN 2.0 models (Figure 1A), with a few advancements: (1) network inference was done with the cMonkey2 biclustering alogirthm $^{4}$; (2) use of TF-target gene interactions with physical binding (ChIP-seq data) ${ }^{17}$ to inform the setenrichment scoring module in cMonkey2; and (3) manually defined condition blocks to subset the gene expression data. The compendium of gene expression data used for training Mtb EGRIN 2.0 was collected from publicly available datasets and previously used to construct the Mtb EGRIN model ${ }^{7}$. Within the expression compendium, transcriptome profiles of $\mathrm{Mtb}$ cultured in conditions of osmotic stress, low iron, carbon monoxide (CO) stress, rifampicin treatment, exposure to lung surfactant or low $\mathrm{pH}$ were represented in $<2 \%$ of the 1900 transcriptomes in the compendium (Figure 1B). However, these condition blocks and all condition blocks were each included in $\sim 20 \%$ of cMonkey2 runs aggregated into EGRIN 2.0 (Figure 1B). Thus, the ensemble-based method of EGRIN 2.0 can effectively up-weight rare conditions and reveal context-dependent regulatory interactions that occur infrequently in the data. We compared the detection of enriched gene sets (Figure 1C) from these rare conditions within biclusters inferred by Mtb EGRIN and 
101

102

103

104

105

106

107

108

109

110

111

112

113

114

115

116

117

118

119

120

121

122

123

124

125

126

127

128

129

130

131

132

133

134

corems identified by Mtb EGRIN 2.0. With the exception of CO stress, corems were significantly better at detecting the enriched gene sets from rare conditions (Figure 1D). It is possible that associations related to CO stress would have been better identified if the condition block was consistently grouped with low oxygen $\left(\mathrm{O}_{2}\right)$ and nitric oxide (NO) stress transcriptomes, as $\mathrm{CO}, \mathrm{O}_{2}$, and $\mathrm{NO}$ are sensed concurrently during Mtb infection ${ }^{18}$. Overall, Mtb EGRIN 2.0 better captures conditional co-regulation of genes from rare environments. Given that low $\mathrm{pH}$ conditions represent only a small portion of the gene expression data, constructing an EGRIN 2.0 model for Mtb was imperative for this study (Figure 1E).

\section{Genome-wide fitness screening identifies corems required for $\mathbf{M}$. tuberculosis adaptation to acidic $\mathbf{p H}$}

To identify the regulatory networks involved in $\mathrm{Mtb}$ adaptation to acidic $\mathrm{pH}$, we utilized transposon mutagenesis coupled with next-generation sequencing (Tn-seq) ${ }^{19}$. Transposon mutant libraries containing $\sim 100 \mathrm{~K}$ individual transposon mutants were generated in $\mathrm{Mtb}$ and cultured in either $\mathrm{pH} 7.0$ or $\mathrm{pH} 5.6$ media for 3 days, at which point the surviving bacteria were plated. Transposon gene junctions were amplified and sequenced from the recovered bacteria and TRANSIT Tn-seq analysis tool ${ }^{20}$ was used to identify transposon insertion sites and calculate their representation in each condition. We developed new software to quantify, display, and summarize the comparative fitness of genes at acidic $\mathrm{pH}$ versus neutral $\mathrm{pH}$ (see Methods, Dataset S1). The corem fitness at acidic pH was then calculated by averaging the conditional change in fitness scores for the genes in each corem (Dataset S2). This analysis revealed corem 71 as being significantly enriched with genes having decreased fitness at acidic $\mathrm{pH}$ (permuted Benjamini-Hochberg, $\mathrm{BH}$, corrected $p$-value $<0.001)$. We performed another Tn-seq fitness screen in the presence and absence of the cell wall-perturbing detergent sodium dodecyl sulfate (SDS). There was no significant decrease in average fitness for corem 71 genes in the presence of SDS (Figure S1, Dataset S1 and S2), suggesting that corem 71 is enriched in genes with reduced fitness specific to acidic $\mathrm{pH}$.

\section{MtrA regulates corem 71 genes that are required for $M$. tuberculosis adaptation to acidic pH}

To find evidence of corem 71 regulation by specific TFs, we investigated the EGRIN 2.0-predicted organization of GREs in the promoters of corem 71 genes. EGRIN 2.0 predicts the frequency of GRE alignment to each genomic position, thus predicting the organization of GREs in gene promoters at nucleotide resolution. EGRIN 2.0 predicts that five GREs are significantly enriched in the promoters of corem 71 genes, with GRE \#11 being the most significant $\left(\mathrm{BH}\right.$ corrected $p$-value $\left.=8.8 \times 10^{-11}\right)$ and found in the promoter of all corem 71 genes with reduced fitness at acidic $\mathrm{pH}$ (Figure 2A). We compared the motif of GRE \#11 to motifs that were deciphered through analysis of ChIP-seq mapped TF binding locations ${ }^{17}$. GRE \#11 significantly matched ( $p$-value $\left.<0.01\right)$ the sequence motif of MtrA $(\operatorname{Rv} 3246 c)$ determined by ChIP-seq ${ }^{17,21}$. Moreover, MtrA ChIP-seq binding sites were identified in the promoters of 
corem 71 genes, including the genes with reduced fitness at low $\mathrm{pH}$, with statistical significance $(\mathrm{BH}$ corrected $p$-value $<0.0001)$. In addition to the genes with reduced fitness at low $\mathrm{pH}$, corem 71 contains 46 genes including the TF, MtrA (Figure 2B). MtrA is the response regulator of a two-component system (TCS) with MtrB. MtrAB is essential for in vitro growth of $\mathrm{Mtb}^{22,23}$, and predicted to be essential for Mtb survival in macrophages ${ }^{24}$ and $\mathrm{Mtb}$ growth in mouse spleen $^{25}$ as determined by Tn-mutagenesis based investigations. Overexpression of MtrA has no effect in vitro but results in decreased growth in macrophages ${ }^{26}$, although the mechanism is unknown. Similar to other essential TFs, there is little characterization of MtrA's regulatory function in Mtb. To better characterize MtrA and its potential role in regulation of corem 71 genes and in acidic $\mathrm{pH}$, we used recent advances in mycobacterial CRISPRinterference (CRISPRi) ${ }^{27}$ to knockdown the expression of $m t r A$. We designed five small guide RNAs (sgRNAs) targeting $m$ trA in Mtb that achieved varying levels of gene knockdown with the CRISPR1 Cas9 from Streptococcus thermpohilus (Table S1) ${ }^{27}$. Two of the Mtb CRISPRi strains (with sgRNA2 and sgRNA3) reached high-level mRNA repression upon the addition of $100 \mathrm{ng} / \mathrm{mL}$ anhydrotetracycline (ATc) and resulted in 3-log viability reduction of Mtb (Figure S2A), compared to controls plated without ATc. With ATc induction, we also observed very limited growth and severe cell aggregation in broth culture (Figure S2B). The other three CRISPRi strains (with sgRNA4, sgRNA5, sgRNA6) achieved lesser degree of $m$ tr $A$ knockdown and viability reduction upon ATc addition (Figure S3A). These CRISPRi strains, with partial knockdown of $m$ trA, were able to grow in broth culture in the presence of ATc, although still with distinct growth inhibition compared to no ATc controls (Figure S3B). The panel of sgRNAs allowed us to tune $m \operatorname{tr} A$ knockdown and obtain strains with a range of growth properties for further characterization studies. Using sgRNA2 and sgRNA3, which produced high-level knockdown of $m$ tr $A$, we collected samples four days after ATc addition for gene expression profiling by RNA sequencing (RNA-seq). We performed differential expression analysis with respect to uninduced (-ATc) controls and confirmed that CRISPRi knockdown of mtrA repressed the expression of many corem 71 genes (Figure 2C, Dataset S3). In particular, all of the corem 71 genes with reduced fitness at acidic $\mathrm{pH}$ were significantly repressed ( $p$-value $<0.01$ ) upon mtrA knockdown (Table 1). Interestingly, the isoniazid induced genes (iniBAC) were significantly up-regulated when $m$ tr $A$ expression was silenced (Figure 2C). The iniBAC genes are induced by a broad range of cell wall biosynthesis inhibitors ${ }^{28}$ and points toward defects in the cell wall when $m$ tr $A$ is silenced.

Given the sequence similarity of MtrA between M. smegmatis (Msm), a non-pathogenic fastgrowing mycobacterium, and Mtb, we sought to also characterize mtrA knockdown in Msm. We generated CRISPRi strains that achieved equally strong repression of mtrA in Msm upon ATc addition. This repression of $m$ trA resulted in a moderate (but significant) growth inhibition of Msm (Figure S4), supporting a recent study that found $m t r A$ is not essential in Msm, in contrast with the Mtb ortholog ${ }^{21}$. We 
performed RNA-seq and analyzed differential expression of all genes due to ATc-induced repression of mtrA in the Msm CRISPRi strain (Figure S5, Dataset S4). Similar to Mtb, the knockdown of $m t r A$ in Msm also resulted in significant repression of the orthologous genes with reduced fitness at low $\mathrm{pH}$ (Table 1), with the exception of the Rv2525c homolog (MSMEG_6815). Of note in the Msm mtrA knockdown strain, was significantly high expression of genes encoding the peptidoglycan (PG) transglycosylases known as resuscitation promoting factors, $r p f B\left(M S M E G \_5249, \log 2\right.$ fold-change $=2.47, p$-value $<0.01$ ) and $r p f E$ (MSMEG_4643, $\log 2$ fold-change $=2.47$ and 5.11, respectively, $p$-value $<0.01$ ). RpfB and RpfE are known to interact with Rpf interacting protein, RipA, in Msm, where the protein complex hydrolyzes PG during cell division ${ }^{29-31}$. RipA is found in Corem 71, had reduced fitness in low $\mathrm{pH}$, and was significantly repressed upon $m$ tr $A$ knockdown (in both $\mathrm{Mtb}$ and Msm). It is possible that increased expression of the $r p f s$ (via unknown regulatory mechanism) could sustain PG cleavage in Msm when ripA is repressed by MtrA. In contrast, $r p f B$ and $r p f E$ were not significantly differentially expressed upon $m t r A$ knockdown in Mtb. The interaction of RipA and Rpfs, or specifically the lack of interaction in Mtb needs further investigation, and could contribute to the difference in MtrA essentiality between Msm and Mtb. These results suggest that while the regulatory activity of MtrA is largely conserved from Msm to Mtb, subtle differences in the function of this TF that has emerged upon their evolutionary divergence has manifested as a particular vulnerability for the human pathogen.

\section{MtrA regulon of $M$. tuberculosis is repressed at low $\mathrm{pH}$}

To examine the transcriptional response of the MtrA regulon (defined as genes in Table 1) at low pH, we grew wild type Mtb to mid-log phase in 7H9 growth medium at neutral $\mathrm{pH}$, washed, then diluted the cells in either neutral or low $\mathrm{pH}$ media (7H9-rich media supplemented with $0.05 \%$ tyloxapol and adjusted to $\mathrm{pH}$ 7.0 or $\mathrm{pH}$ 5.6). Following transition to either neutral or acidic media, we monitored growth for 7 days and collected samples at $24 \mathrm{~h}$ for transcriptome profiling by RNA-seq. As expected, Mtb growth was stalled at acidic pH (Figure 3A). We also observed a significant decrease in MtrA regulon expression at acidic $\mathrm{pH}$ compared to neutral pH (Figure 3B). This change in expression of MtrA regulon genes, along with the Tnseq data demonstrating their essentiality at low $\mathrm{pH}$, suggests that reducing but maintaining expression of the regulon genes is important for Mtb's survival in acidic conditions.

Given that the mtrA knockdown strains were growth attenuated and would be further growth inhibited in low $\mathrm{pH}$ media, we reasoned that one approach to understand MtrA perturbation at acidic $\mathrm{pH}$ would be to overexpress $m t r A$. Using the ATc-inducible overexpression strain previously described ${ }^{17}$, we repeated the transfer of mid-log phase cells to either neutral media or low $\mathrm{pH}$ media. After transfer, we induced $m t r A$ expression with ATc and collected cells at $18 \mathrm{~h}$ for transcriptional profiling by RNA-seq. Addition of ATc resulted in six-fold increase in $m t r A$ expression relative to uninduced at neutral $\mathrm{pH}$ and 
four-fold increase in $m t r A$ expression at acidic $\mathrm{pH}(p$-value $<0.001)$. While the expression of the MtrA regulon was not affected by overexpression of $m \operatorname{tr} A$ at neutral $\mathrm{pH}$, it was significantly downregulated at acidic $\mathrm{pH}$ (Figure 3C). Together, these results suggest that overexpression of $m t r A$ during macrophage infection, when Mtb also experiences acid stress, interferes with optimal MtrA levels and possibly its phosphorylation by $\mathrm{MtrB}^{26}$, resulting in severe repression of its downstream target genes. These results explain previous observations that $m t r A$ overexpression had no effect on in vitro growth of Mtb, but abolished its ability to replicate in THP-1 macrophages ${ }^{26}$.

\section{MtrA knockdown results in $M$. tuberculosis and $M$. smegmatis growth defects}

212 As mentioned above, among the regulatory targets of MtrA (Table 1) are four genes with PG hydrolytic activity. These genes, ripA, ripD, Rv2525c and amil, encode enzymes that are capable of cleaving PG and contribute to the physical separation of two daughter cells during cell division. RipA is a D,L-endopeptidase, cleaving PG within the peptide stem $^{32}$. RipD also contains an endopeptidase NlpC/p60 domain similar to RipA, but the purified protein demonstrated a non-catalytic PG-binding function ${ }^{33}$. Structural studies suggest a PG glycoside hydrolase function for Rv2525c, although its activity has not been established ${ }^{34}$. Finally, Amil contains an amidase_3 domain that cleaves the peptide stem from $\mathrm{N}$-acetyl-muramic acid on the PG glycan backbone ${ }^{35}$. To assess the effect of MtrA regulation on these PG cleavage enzymes and cell division, we used the fluorescent $D$-alanine analogue HADA, which incorporates into newly synthesized PG. We fluorescently labeled the PG of Mtb mtrA knockdown cells containing sgRNA5, which achieved

222 low-level mtrA repression, and observed elongated multiseptated cells (Figure 4A). Cells with ATcinduced $m \operatorname{tr} A$ knockdown had an increased median cell length and a larger variation in cell length compared to uninduced cells (Figure 4B). Nearly all $(\sim 75 \%)$ of the $m$ trA knockdown cells contained at least one septum, while septa were present in only $15 \%$ of the uninduced cells (Figure 4C). In fact, more than one septum were present in a majority of the $m \operatorname{tr} A$ knockdown cells, indicating that septa form but the cells fail to divide. Interestingly, the mtrA knockdown cells adopted an abnormal curved or bent shape at the poles, which was not found in the uninduced cells (Figure 4A). As PG is vital for maintaining cell shape ${ }^{36}$, the curved shape phenotype could be a result of a disrupted PG layer in growing cells. In mycobacteria, growth and division are uncoupled events and the organism continues to grow regardless of the occurrence of cell division $^{37}$. During cell growth, PG cleavage (via PG hydrolases) may be important to allow for incorporation of new PG at the cell poles ${ }^{38}$. It is interesting to speculate that repression of the PG hydrolases 
(MSMEG_6281) were significantly repressed in the Msm mtrA knockdown strain (Table 1), the Rv2525c homolog (MSMEG_6815) was not. This indicates similar, but also unique, roles of MtrA in regulating PG cleavage between Msm and Mtb.

\section{MtrA knockdown increases the susceptibility of $M$. tuberculosis to frontline antibiotics}

242 A consequence of defective cell division and growth could be altered susceptibility to antitubercular drugs.

243 We used our Mtb CRISPRi strain with sgRNA5 to determine whether the bactericidal activities of frontline

244 TB drugs are altered when $m t r A$ expression is moderately repressed. We compared time-kill curves for the

$245 m t r A$ knockdown strain induced with ATc versus uninduced when exposed to sub MIC concentrations of

246 isoniazid and rifampicin. Isoniazid is a frontline antitubercular drug that rapidly kills actively dividing

247 bacilli, whereas rifampicin is a sterilizing drug that kills both replicating and persistent bacilli ${ }^{39,40}$. The two

248 drugs have different mechanisms of action and different chemical properties, yet at concentrations that only

249 partially inhibited growth in the uninduced controls, both drugs were able to completely sterilize the Mtb mtrA knockdown cultures (Figure 5A). There were different drug susceptibility patterns observed in Msm $m t r A$ knockdown cultures, as measured by disk diffusion (Figure S7A). We found that Msm $m$ trA knockdown cultures showed increased susceptibility to rifampicin, carbinicillin and vancomycin. We also tested cycloserine and isoniazid, but saw no difference in susceptibility compared to the control. The results for the Msm mtrA knockdown demonstrated a pattern of increased susceptibility to higher molecular weight antibiotics. Larger molecules may more easily enter the Msm mtrA knockdown cells due to alterations in cell wall composition. To further investigate the changes in drug susceptibility in Msm, we tested the cell wall permeability of the Msm mtrA knockdown cells using an ethidium bromide (EtBr) diffusion assay and found $m t r A$ repression resulted in reduced EtBr diffusion compared to control (Figure S7B). This suggests that the mechanism for increased susceptibility to high molecular weight antibiotics in Msm is not simply due to increases in cell wall permeability. Other drug transport mechanisms such as porin-like proteins may be affected in the Msm $m$ trA knockdown cell.

\section{DISCUSSION}

The EGRIN 2.0 model for Mtb reveals, with unprecedented detail, how the pathogen tailors transcriptional responses to survive stresses experienced within the host. The model captures the genome-wide distribution of GREs within each promoter and statistically significant gene associations in corems from host-relevant conditions. The ensemble network inference methodology allows detection of regulatory events for conditions that represent only a small portion of the entire data set, such as acidic $\mathrm{pH}$, a stress Mtb faces in 
the maturing macrophage phagosome. We performed extensive experiments to validate a model prediction that genes controlled by the MtrAB regulatory circuit are essential for Mtb at acidic $\mathrm{pH}$. We demonstrated that MtrA regulation of PG cleavage is required for Mtb cell division. Further, we showed that even weak repression of $m t r A$ results in significant growth inhibition and completely clears the bacteria with low-dose isoniazid or rifampicin treatment.

Mtb has successfully exploited macrophages as its primary niche in vivo, evolving the capability to withstand a myriad of stresses with the macrophage, including acid stress. Several proteins have been implicated in Mtb's response to acid stress, such as the two-component system PhoPR ${ }^{15,16,41}$, the outer membrane protein OmpATB ${ }^{42}$, and the PG hydrolase RipA ${ }^{43}$. Our work also suggests that PG cleavage by MtrA-regulated PG hydrolases is necessary for Mtb to reside in the acidic phagolysosome. The essentiality of PG cleavage during acid stress points towards residual cell division or PG hydrolysis for the incorporation of new PG, even when Mtb growth is reduced. It has been proposed that replication during stress may favor bet-hedging by distributing damaged macromolecules between progeny cells, thus creating heterogeneous subpopulations with altered fitness and the capacity to survive stress ${ }^{44,45}$. Alternatively, the PG cleavage enzymes may permit PG rearrangement or repair, which could lead to cell wall changes for nutrient uptake $\mathrm{e}^{46}$ or the maintenance of $\mathrm{pH}$ homeostasis ${ }^{47}$ during acid stress.

The maturing macrophage phagosome acquires bactericidal properties, such as acidic $\mathrm{pH}$, to restrict and kill invading pathogens. However, it has been shown that during infection in mice, some macrophage lineages control Mtb better than others ${ }^{48-50}$. Analysis of Mtb's transcriptional response from alveolar macrophages, which promote Mtb growth, and monocyte derived macrophages, that restrict Mtb growth ${ }^{51}$, finds that the MtrA regulon is significantly down-regulated in monocyte derived macrophages compared to alveolar macrophages ( $\log 2$ fold-change $=-1.1, p$-value $\left.=6.76 \times 10^{-5}\right)$. This suggests that monocyte derived macrophages contain acidified phagolysosomes that trigger MtrA-mediated repression of PG cleavage. This also supports the notion that monocyte derived macrophages may be innately more capable of restricting bacterial growth compared to alveolar macrophages, since repression of mtrA and target genes would also inhibit cell division. We propose that inhibiting MtrA could restrict Mtb growth in both macrophage populations and promote clearance from the host.

Our study reveals that Mtb cell division, particularly PG cleavage, is transcriptionally regulated by MtrA. We have demonstrated that the MtrA regulon includes multiple PG hydrolases, selected for coregulation by evolution. Interestingly, corem 71 includes three other PG remodeling enzymes (dacB1 (Rv3330), penicillin-binding lipoprotein (Rv2864c), and the amidase, ami3 (Rv3811)), that were not differentially expressed with $m$ trA knockdown in Mtb. This demonstrates how corems group together genes that are functionally related even though their co-regulation is mediated by different mechanisms ${ }^{1}$. Despite their similar enzymatic functions, the PG cleavage enzymes controlled by MtrA could fulfill individually 
important roles in specific conditions. Recent work established the contextual redundancy of the MtrA regulatory targets, ripA and amil in $\mathrm{Mtb}^{52}$. The authors showed that Amil sustains in vitro cell division in cells lacking ripA, and vice versa, but that this redundancy is not sufficient for replication and persistence of Mtb in the host ${ }^{52}$. While we demonstrated that MtrA coordinates the transcription of both PG cleavage genes (along with ripD and $R v 2525 c$ ), the conditional nature of their redundancy could be related to interacting proteins that may be associated with specific conditional or temporal events during infection. Regardless of their individual roles, their coordinated regulation offers unique opportunity to target multiple PG modifying enzymes by inhibiting MtrA with a drug. In fact, small molecules have been identified that inhibit the DNA binding activity of $\mathrm{MtrA}^{53}$. The compounds were found to inhibit the expression of $m t r A$ and restrict Mtb replication in vitro and within macrophages. As such, there exists chemical space for the development of potent MtrA inhibitors.

Earlier studies proposed a handful of MtrA regulatory targets (dnaA, ripA, fbpB, ftsI and wag31) using Msm or Mtb strains overproducing wild-type and mutant MtrA ${ }^{21,26,54,55}$. Our model and experimental results confirmed the previously reported MtrA targets, ripA and wag31. Additionally, several recent studies have evaluated MtrA regulatory targets using global ChIP-seq analysis with Mtb strains overexpressing C-terminal FLAG-MtrA ${ }^{17}$, N-terminal His-tagged $\mathrm{MtrA}^{56}$, or proposed phosphorylation competent MtrA protein (replacement of tyrosine with cysteine at position 102) ${ }^{21}$. Among the 14 putative MtrA targets identified across these studies (Table S2), we observed significant differential expression ( $p$ value $<0.01$ ) with Mtb mtrA knockdown of the conserved protein Rv1815 (log2 fold-change = -1.9) and the resuscitation promoting factors $r p f A$ ( $\log 2$ fold-change $=-1.9)$, and $r p f C(\log 2$ fold-change $=-2.5)$. Interestingly, the $r p f A$ and $r p f C$ protein products also have predicted $\mathrm{PG}$ cleavage activity ${ }^{57,58}$. Additionally, there was significant repression of $\operatorname{ripB}(R v 1478, \log 2$ fold-change $=-3.4)$, an PG endopeptidase with activity similar to $\operatorname{rip}^{32}$, upon $m \operatorname{tr} A$ knockdown in Mtb. While these PG hydrolases are not part of corem 71 and may not be important for PG cleavage during infection in the host, they could be regulated by MtrA and active in other conditions. This points to another capability of EGRIN 2.0, to decipher how regulation by a TF varies conditionally across different targets.

Importantly, this study is the first to characterize MtrA loss directly in Mtb. Previous attempts to construct a $\triangle m t r A$ mutant in Mtb were unsuccessful ${ }^{21,59,60}$, but the CRISPRi platform for mycobacteria allowed easy manipulation of the essential gene ${ }^{27}$. Using CRISPRi to repress $m t r A$ in Mtb and profile genome-wide transcriptional changes, we have validated new regulatory targets of the essential response regulator (Table 1). Moreover, we have conducted parallel experiments in Msm and found conserved MtrA regulatory targets between the species. However, there were also key differences in PG cleavage genes. (e.g., $R v 2525 c, r p f B, r p f E$ ). These results, along with the important difference in MtrA essentiality between Msm and Mtb calls into question using the fast-growing mycobacterial model organism to interpret MtrA 
activity in Mtb. This caution may extend to the study of all mycobacterial PG remodeling enzymes, as there are fundamental differences in PG incorporation ${ }^{61}$ and PG hydrolase activity ${ }^{52,62}$ between Msm and Mtb. These findings propose evolution from saprophyte to pathogen has occurred, in part, through regulatory networks and PG remodeling activities to function in the host environment.

Using sgRNAs of various "strengths", we were able to tune the magnitude of $m$ tr $A$ knockdown and establish that even minimal mtrA knockdown is lethal to Mtb. All bacteria need to coordinate synthesis and breakdown of PG to maintain integrity through cell division; the importance of this is evident from the number of PG-targeting antibiotics. However, the complexity of the Mtb cell wall represents a distinct challenge to the pathogen, requiring specialized mechanisms for cell division and growth to occur ${ }^{63}$. We propose the unique cell wall properties of Mtb and the regulation of multiple PG cleavage enzymes contributes to the extreme vulnerability of MtrA in Mtb. Furthermore, we demonstrated that $<10 \%$ inhibition via mtrA knockdown, resulted in complete clearance of Mtb from cultures with sublethal doses of drugs currently used to treat TB. Ultimately, our work presents MtrA as an attractive therapeutic target with several advantages: (1) inhibition of MtrA potentiates frontline drugs, requiring lower therapeutic doses; (2) inhibition of MtrA restricts bacterial growth, especially within host environments permissive for varying degrees of growth; and (3) chemical space already exists ${ }^{53}$, making MtrA a tractable target. We hope that deciphering conditional gene regulatory networks in the Mtb EGRIN 2.0 model will identify additional drug targets and treatment-enhancing strategies to better control TB.

Acknowledgements. We gratefully acknowledge Sarah Fortune for kindly providing us with the PLJR962 and PLJR965 plasmids. We thank Michael VanNieuwenhze for kindly providing HADA. We thank members of the Baliga lab for critical discussions and feedback. This work is funded by the National Institute of Allergy and Infectious Diseases of the National Institutes of Health (R01AI128215, R01AI141953 and U19AI135976) and the Bill and Melinda Gates Foundation (INV-009322).

Competing interest. The authors declare no competing interest.

Author Contributions. EJRP and NSB conceived the study. ANB and DJR ran cMonkey and constructed the ensemble. EJRP analyzed the ensemble and all data represented. WW provided assistance with ensemble analyses and Web portal. RM analyzed Tn-seq data. ST developed the Web portal. EJRP designed and supervised experimental validations. AK, VS, WC, MP and RR performed experiments. MLAO helped with computational analyses related to rare co-regulation events. $\mathrm{AB}$ helped with designing experimental

371 approved its content. 
373 Data availability. The data reported in this paper are available in Supplementary Datasets 1-5. EGRIN 2.0

374 code is available in Baliga lab GitHub (https://github.com/baliga-lab/egrin2-tools) with additional

375 information on the supporting Web site (http://egrin2.systemsbiology.net). All corem and GREs of the Mtb

376 EGRIN 2.0 model are available on the Mtb web portal: http://networks.systemsbiology.net/mtb/. The raw

377 Tn-seq fastq sequence data files are deposited in the Sequence Read Archive database under accession

378 PRJNA701946. The RNA-seq data generated for this study are available in the Gene Expression Omnibus

379 under accession no. GSE166806.

\section{METHODS}

EGRIN 2.0 construction. EGRIN 2.0 was constructed as an ensemble of many individual EGRIN models ( $\sim 300$ for M. tuberculosis). Each EGRIN model was constructed using cMonkey $2^{4}$. The input to $c$ Monkey2 was 1,861 transcriptome profiles with metadata collected about each experiment to annotate the environmental context (termed condition block, Dataset S5). Other input to cMonkey2 were upstream regions of all genes, and functional association networks, including operon predictions from MicrobesOnline $^{64}$ and functional protein interactions from EMBL String databases ${ }^{65}$. We integrated the EGRIN models and mined the ensemble to discover frequently reoccurring features and associations as described previously ${ }^{1}$. We refer to the modules detected by our procedure as co-regulated modules, or corems, the frequently re-occurring de novo cis-regulatory motifs as gene regulatory elements, or GREs. Full description of the algorithms and each post-processing step is documented in Supplementary Information of Brooks et al ${ }^{l}$.

Bacterial strains. All M. tuberculosis strains are derivatives of H37Rv; all M. smegmatis strains are derivatives of $\mathrm{mc}^{2} 155$.

397 Media. M. tuberculosis and M. smegmatis were grown at $37^{\circ} \mathrm{C}$ in Middlebrook $7 \mathrm{H} 9$ broth or $7 \mathrm{H} 10$ plates 398 supplemented with $0.2 \%$ glycerol, $0.05 \%$ Tween-80, and 10\% ADC (liquid media) or OADC (plates), with 399 aeration. Where indicated, anhydrotetracycline (ATc) was used at $100 \mathrm{ng} / \mathrm{ml}$. For comparison of neutral 400 and acidic $\mathrm{pH}$ media, the neutral media was $7 \mathrm{H} 9$ broth supplemented with $0.2 \%$ glycerol, $0.05 \%$ tyloxapol, 401 and ADC buffered with $100 \mathrm{mM} 3-(\mathrm{N}$-morpholino)propanesulfonic acid (MOPS) to $\mathrm{pH}$ 7. The acidic $\mathrm{pH}$ 402 media was the same 7H9-rich media buffered with $100 \mathrm{mM}$ 2-(N-morpholino)ethanesulfonic acid (MES) 
0.7), washed in 1x PBS three times, and diluted into either neutral or acidic $\mathrm{pH}$ media at a starting density of $\mathrm{OD}_{600}=0.05$.

Transposon mutant library sequencing. Transposon mutant libraries were constructed using the $\phi$ MycoMarT7 phagemid in M. tuberculosis H37Rv. The mutant libraries were grown to mid-log phase in 7H9-rich media, then diluted to $\mathrm{OD}_{600}=0.1$ in 7H9-rich media at neutral $(\mathrm{pH} 7.0)$ or acidic $\mathrm{pH}(\mathrm{pH}$ 5.6) media. In another experiment, the mutant libraries were diluted back in 7H9-rich media with or without $0.05 \%$ SDS. Following dilution, mutant samples were diluted in PBS with $0.05 \%$ Tween-80 and plated onto $245 \mathrm{~mm}$ x $245 \mathrm{~mm} 7 \mathrm{H} 10$ plates supplemented with kanamycin $(50 \mu \mathrm{g} / \mathrm{ml})$ to T0. Additional samples were exposed to the stressors for 72 hours and then plated. Colonies grew for 21 days and were collected

413 for genomic DNA isolation. The mutant composition was determined by sequencing amplicons of the transposon-genome junctions following the protocol outlined by Long et al ${ }^{66}$. Paired-end reads were run on an Illumina HiSeq 2500 at the Genomics Services Core at Fred Hutchinson Cancer Research Center. Mapping and quantification of transposon insertions sites was done using TRANSIT analysis platform ${ }^{20}$.

417 The change in fitness between Day 3 and T0 for all genes was determined following the strategy of van Opijnen et al. ${ }^{67}$ using a custom processing pipeline, full description and code is available at http://networks.systemsbiology.net/mtb/. The raw Tn-seq fastq sequence data files are deposited in the Sequence Read Archive database under accession PRJNA701946.

Permutation test for evaluating significance of overlap between corems and genes with reduced fitness.

422 The genes with reduced delta fitness in stress (either acidic pH or SDS treatment) were permutated 1000

423 times to generate shuffled gene clusters. In each permutation, the produced shuffled gene clusters had the

424 same size as corem 71. Then, the average delta fitness for each shuffled gene set was computed and

425 compared to the average delta fitness for corem 71 . The overall permutation test $p$-value was computed as

426 the proportion of cases (out of 1000 permutations) in which the average delta fitness was equal or lower

427 than the observed value in corem 71.

Gene expression profiling of CRISPRi-mediated mtrA knockdown. For each sample, cultures were grown to mid-log phase in 7H9-rich media with Kanamycin $(50 \mu \mathrm{g} / \mathrm{ml})$ and then diluted back in the presence or absence of ATc to induce CRISPRi-mediated mtrA knockdown. Knockdown was allowed to proceed for 14 hours (M. smegmatis) or 4 days (M. tuberculosis). Samples, in biological quadruplicate, were collected by centrifugation at high speed for $5 \mathrm{~min}$, discarding supernatant and immediately flash freezing the cell pellet in liquid nitrogen. Cell pellets were stored at $-80^{\circ} \mathrm{C}$ until RNA extraction was performed as previously described ${ }^{41}$. The processing of samples for sequencing and read alignment is described below. 
The RNA-seq data profiling CRISPRi-mediated mtrA knockdown generated for this study are publicly available from the Gene Expression Omnibus at GSE166806.

Gene expression profiling of mtrA overexpression. To investigate gene expression changes of mtrA overexpression, we used M. tuberculosis strain containing an ATc-inducible vector as described previously ${ }^{17}$. For each sample, cultures of M. tuberculosis were cultured in standard 7H9-rich media containing $50 \mu \mathrm{g} / \mathrm{ml}$ hygromycin B to mid-log phase, washed three times in 1x PBS and diluted back to $\mathrm{OD}_{600}=0.05$ into either neutral or acidic $\mathrm{pH}$ media with $50 \mu \mathrm{g} / \mathrm{ml}$ hygromycin $\mathrm{B}$ and the presence or absence of ATc inducer. Samples, in biological triplicate were collected after 18 hours of ATc induction, centrifuged at high speed for 5 min, supernatant was discarded and the cell pellet was immediately flash frozen in liquid nitrogen. Cell pellets were stored at $-80^{\circ} \mathrm{C}$ until RNA extraction was performed as previously described ${ }^{41}$. The processing of samples for sequencing and read alignment is described below. The RNA-seq data profiling $m t r A$ overexpression in netural and acidic $\mathrm{pH}$ generated for this study are publicly available from the Gene Expression Omnibus at GSE166806.

Gene expression profiling at netural or acidic $p H$. To investigate gene expression changes of $M$. tuberculosis at acidic $\mathrm{pH}$, cultures of M. tuberculosis were cultured in standard 7H9-rich media to mid-log phase, washed three times in $1 \mathrm{x}$ PBS and diluted back to $\mathrm{OD}_{600}=0.1$ into either neutral or acidic $\mathrm{pH}$ media. Samples, in biological triplicate, were collected right after transfer and after 24 hours in neutral or acidic $\mathrm{pH}$ media. Samples were centrifuged at high speed for $5 \mathrm{~min}$, supernatant was discarded and the cell pellet was immediately flash frozen in liquid nitrogen. Cell pellets were stored at $-80^{\circ} \mathrm{C}$ until RNA extraction was performed as previously described ${ }^{41}$. The processing of samples for sequencing and read alignment is described below. The RNA-seq data profiling $m t r A$ overexpression in netural and acidic $\mathrm{pH}$ generated for this study are publicly available from the Gene Expression Omnibus at GSE166806.

Processing and analysis of RNA-seq data. Sample collection and RNA-extraction was performed as described above. Total RNA samples were depleted of ribosomal RNA using the Ribo-Zero Bacteria rRNA Removal Kit (Illumina, San Diego, CA). Quality and purity of mRNA samples was determined with 2100 Bioanalyzer (Agilent, Santa Clara, CA). Samples were prepared with TrueSeq Stranded mRNA HT library preparation kit (Illumina, San Diego, CA). All samples were sequenced on the NextSeq sequencing instrument in a high output 150 v2 flow cell. Paired-end 75 bp reads were checked for technical artifacts using Illumina default quality filtering steps. Raw FASTQ read data were processed using the R package DuffyNGS $^{42}$. Briefly, raw reads were passed through a 2-stage alignment pipeline: (i) a pre-alignment stage to filter out unwanted transcripts, such as rRNA; and (ii) a main genomic alignment stage against the genome of interest. Reads were aligned to M. tuberculosis H37Rv (ASM19595v2) or M. smegmatis mc 155 
(ASM1500v1) with Bowtie $2^{43}$, using the command line option "very-sensitive." BAM files from stage (ii) were converted into read depth wiggle tracks that recorded both uniquely mapped and multiply mapped reads to each of the forward and reverse strands of the genome(s) at single-nucleotide resolution. Gene transcript abundance was then measured by summing total reads landing inside annotated gene boundaries,

472 expressed as both RPKM and raw read counts. All RNA-seq data (raw and processed data) generated for

473 this study are publicly available at the Gene Expression Omnibus under accession numbers GSE166806.

Differential expression analysis. We used a panel of 5 DE tools compiled in DuffyNGS to identify gene expression changes as previously described. The tools included (i) RoundRobin (in-house); (ii) RankProduct $^{68}$; (iii) significance analysis of microarrays (SAM) ${ }^{69}$; (iv) EdgeR ${ }^{70}$; and (v) DESeq2 ${ }^{71}$. Each DE tool was called with appropriate default parameters and operated on the same set of transcription results, using RPKM abundance units for RoundRobin, RankProduct, and SAM and raw read count abundance units for DESeq2 and EdgeR. Each DE tool's explicit measurements of differential expression (foldchange) and significance ( $p$-value) were similarly combined via appropriate averaging (arithmetic and geometric mean, respectively).

Peptidoglycan labeling with fluorescent d-alanine analogues. Fluorescent d-alanine analog (FDAA) HADA (HCC-amino-d-alanine) was synthesized by Michael VanNieuwenhze at Indiana University using methods previously published ${ }^{72}$. Cultures were grown to mid-log phase in 7H9-rich media with Kanamycin $(50 \mu \mathrm{g} / \mathrm{ml})$ and then diluted back in the presence or absence of ATc to induce CRISPRi-mediated $m t r A$ knockdown. Knockdown was allowed to proceed for 14 hours (M. smegmatis) or 4 days (M. tuberculosis). Cultures were then inoculated at an OD600 of 0.1 to 0.3 into 7H9 medium supplemented with 1 mM HADA and incubated for 3.5 hours (M. smegmatis) or 20 hours (M. tuberculosis). Bacterial suspension were washed 3 times with PBS- $0.05 \%$ Tween- 80 and fixed with paraformaldehyde for 30 min (M. smegmatis) or 4 hours (M. tuberculosis), to ensure bacterial death for further imaging outside a contained environment.

Super-resolution microscopy. Fixed bacterial suspensions were mixed with the same volume of mounting medium and $10 \mu \mathrm{l}$ amounts were spread on microscope slides and covered with cover glasses. Microscopy imaging was performed using SP8 Lightning super-resolution microscope (Leica Microsystems). Images were analyzed using ImageJ software.

Phenotyping CRISPRi-mediated mtrA knockdown. To visually monitor the effects of CRIPSRI-mediated 496 mtrA knockdown, cultures were grown to mid-log phase in 7H9-rich media with Kanamycin $(50 \mu \mathrm{g} / \mathrm{ml})$ 497 and spotted on solid media with or without Atc. Plates were incubated at $37^{\circ} \mathrm{C}$ for 21 days (M. tuberculosis). 

M. smegmatis was spread on LB agar plates containing $100 \mathrm{ng} / \mathrm{ml}$ anhydrotetracycline inducer. A filter disc with $10 \mu \mathrm{l}$ of carbenicillin $(100 \mathrm{mg} / \mathrm{ml})$, isoniazid $(0.5 \mathrm{mg} / \mathrm{ml})$, vancomycin $(6 \mathrm{mg} / \mathrm{ml})$, cycloserine $(100$ $\mathrm{mg} / \mathrm{ml})$ or rifampicin $(0.5 \mathrm{mg} / \mathrm{ml})$ was placed in the center of plate and the diameter of inhibition of growth was measured after 4 days of growth. media with Kanamycin $(50 \mu \mathrm{g} / \mathrm{ml})$ and then diluted back in the presence or absence of ATc to induce

506 CRISPRi-mediated $m t r A$ knockdown. Knockdown was allowed to proceed for 4 days. Antibiotic (0.18 $507 \mu \mathrm{g} / \mathrm{ml}$ isoniazid or $0.006 \mu \mathrm{g} / \mathrm{ml}$ ) or vehicle control was added to the cultures and samples were serially 508 diluted and plated on 7H10 agar plates. Samples were taken for 7 days following antibiotic treatment and colonies were counted after 21 days. All time-kill assays were performed in biological triplicate.

\section{References}

5121 Brooks, A. N. et al. A system-level model for the microbial regulatory genome. Mol Syst Biol 10, $513 \quad$ 740, doi:10.15252/msb.20145160 (2014).

5142 Bonneau, R. et al. A predictive model for transcriptional control of physiology in a free living cell. Cell 131, 1354-1365, doi:10.1016/j.cell.2007.10.053 (2007).

3 Reiss, D. J., Baliga, N. S. \& Bonneau, R. Integrated biclustering of heterogeneous genome-wide datasets for the inference of global regulatory networks. BMC Bioinformatics 7, 280, doi:10.1186/1471-2105-7-280 (2006).

4 Reiss, D. J., Plaisier, C. L., Wu, W. J. \& Baliga, N. S. cMonkey2: Automated, systematic, integrated detection of co-regulated gene modules for any organism. Nucleic Acids Res 43, e87, doi:10.1093/nar/gkv300 (2015). (2006).

6 Plaisier, C. L. et al. Causal Mechanistic Regulatory Network for Glioblastoma Deciphered Using Systems Genetics Network Analysis. Cell Syst 3, 172-186, doi:10.1016/j.cels.2016.06.006 (2016).

7 Peterson, E. J. et al. A high-resolution network model for global gene regulation in Mycobacterium tuberculosis. Nucleic Acids Res 42, 11291-11303, doi:10.1093/nar/gku777 (2014).

8 Turkarslan, S. et al. Niche adaptation by expansion and reprogramming of general transcription factors. Mol Syst Biol 7, 554, doi:10.1038/msb.2011.87 (2011).

9 Ashworth, J. et al. Genome-wide diel growth state transitions in the diatom Thalassiosira pseudonana. Proc Natl Acad Sci U S A 110, 7518-7523, doi:10.1073/pnas.1300962110 (2013).

10 Rothchild, A. C. et al. MiR-155-regulated molecular network orchestrates cell fate in the innate and adaptive immune response to Mycobacterium tuberculosis. Proc Natl Acad Sci U S A 113, E6172-E6181, doi:10.1073/pnas.1608255113 (2016). 
652

653

654

655

656

657

658

659

660

661

662

663

664

665

666

667

668

669

670

671

672

673

674

675

676

677

678

679

680

47 Rao, M., Streur, T. L., Aldwell, F. E. \& Cook, G. M. Intracellular pH regulation by Mycobacterium smegmatis and Mycobacterium bovis BCG. Microbiology (Reading) 147, 1017-1024, doi:10.1099/00221287-147-4-1017 (2001).

48 Huang, L., Nazarova, E. V., Tan, S., Liu, Y. \& Russell, D. G. Growth of Mycobacterium tuberculosis in vivo segregates with host macrophage metabolism and ontogeny. J Exp Med 215, 1135-1152, doi:10.1084/jem.20172020 (2018).

49 Peterson, E. J. et al. Path-seq identifies an essential mycolate remodeling program for mycobacterial host adaptation. Mol Syst Biol 15, e8584, doi:10.15252/msb.20188584 (2019).

50 Rothchild, A. C. et al. Alveolar macrophages generate a noncanonical NRF2-driven transcriptional response to Mycobacterium tuberculosis in vivo. Sci Immunol 4, doi:10.1126/sciimmunol.aaw6693 (2019).

51 Pisu, D., Huang, L., Grenier, J. K. \& Russell, D. G. Dual RNA-Seq of Mtb-Infected Macrophages In Vivo Reveals Ontologically Distinct Host-Pathogen Interactions. Cell Rep 30, 335-350 e334, doi:10.1016/j.celrep.2019.12.033 (2020).

52 Healy, C., Gouzy, A. \& Ehrt, S. Peptidoglycan Hydrolases RipA and Ami1 Are Critical for Replication and Persistence of Mycobacterium tuberculosis in the Host. mBio 11, doi:10.1128/mBio.03315-19 (2020).

53 Banerjee, S. K. et al. Targeting multiple response regulators of Mycobacterium tuberculosis augments the host immune response to infection. Sci Rep 6, 25851, doi:10.1038/srep25851 (2016).

54 Rajagopalan, M. et al. Mycobacterium tuberculosis origin of replication and the promoter for immunodominant secreted antigen $85 \mathrm{~B}$ are the targets of MtrA, the essential response regulator. J Biol Chem 285, 15816-15827, doi:10.1074/jbc.M109.040097 (2010).

55 Plocinska, R. et al. Septal localization of the Mycobacterium tuberculosis MtrB sensor kinase promotes MtrA regulon expression. J Biol Chem 287, 23887-23899, doi:10.1074/jbc.M112.346544 (2012).

56 Chatterjee, A. et al. Global mapping of MtrA-binding sites links MtrA to regulation of its targets in Mycobacterium tuberculosis. Microbiology (Reading) 164, 99-110, doi:10.1099/mic.0.000585 (2018).

57 Cohen-Gonsaud, M. et al. The structure of a resuscitation-promoting factor domain from Mycobacterium tuberculosis shows homology to lysozymes. Nat Struct Mol Biol 12, 270-273, doi:10.1038/nsmb905 (2005).

58 Cohen-Gonsaud, M. et al. Resuscitation-promoting factors possess a lysozyme-like domain. Trends Biochem Sci 29, 7-10, doi:10.1016/j.tibs.2003.10.009 (2004).

59 Parish, T. et al. Deletion of two-component regulatory systems increases the virulence of Mycobacterium tuberculosis. Infect Immun 71, 1134-1140, doi:10.1128/iai.71.3.1134-1140.2003 (2003).

60 Robertson, D., Carroll, P. \& Parish, T. Rapid recombination screening to test gene essentiality demonstrates that pyrH is essential in Mycobacterium tuberculosis. Tuberculosis (Edinb) 87, 450-458, doi:10.1016/j.tube.2007.05.015 (2007).

61 Botella, H. et al. Distinct Spatiotemporal Dynamics of Peptidoglycan Synthesis between Mycobacterium smegmatis and Mycobacterium tuberculosis. mBio 8, doi:10.1128/mBio.0118317 (2017).

62 Senzani, S. et al. An Amidase_3 domain-containing N-acetylmuramyl-L-alanine amidase is required for mycobacterial cell division. Sci Rep 7, 1140, doi:10.1038/s41598-017-01184-7 (2017).

63 Kieser, K. J. \& Rubin, E. J. How sisters grow apart: mycobacterial growth and division. Nat Rev Microbiol 12, 550-562, doi:10.1038/nrmicro3299 (2014). 
68164 Dehal, P. S. et al. MicrobesOnline: an integrated portal for comparative and functional

682 genomics. Nucleic Acids Res 38, D396-400, doi:10.1093/nar/gkp919 (2010).

65 Sanz, J. et al. The transcriptional regulatory network of Mycobacterium tuberculosis. PLoS One 6, e22178, doi:10.1371/journal.pone.0022178 (2011).

66 Long, J. E. et al. Identifying essential genes in Mycobacterium tuberculosis by global phenotypic profiling. Methods Mol Biol 1279, 79-95, doi:10.1007/978-1-4939-2398-4_6 (2015).

67 van Opijnen, T., Bodi, K. L. \& Camilli, A. Tn-seq: high-throughput parallel sequencing for fitness and genetic interaction studies in microorganisms. Nat Methods 6, 767-772, doi:10.1038/nmeth.1377 (2009).

68 Breitling, R., Armengaud, P., Amtmann, A. \& Herzyk, P. Rank products: a simple, yet powerful, new method to detect differentially regulated genes in replicated microarray experiments. FEBS Lett 573, 83-92, doi:10.1016/j.febslet.2004.07.055 (2004).

69 Tusher, V. G., Tibshirani, R. \& Chu, G. Significance analysis of microarrays applied to the ionizing radiation response. Proc Natl Acad Sci U S A 98, 5116-5121, doi:10.1073/pnas.091062498 (2001).

70 Robinson, M. D. \& Smyth, G. K. Small-sample estimation of negative binomial dispersion, with applications to SAGE data. Biostatistics 9, 321-332, doi:10.1093/biostatistics/kxm030 (2008).

71 Love, M. I., Huber, W. \& Anders, S. Moderated estimation of fold change and dispersion for RNA-seq data with DESeq2. Genome Biol 15, 550, doi:10.1186/s13059-014-0550-8 (2014).

72 Kuru, E., Tekkam, S., Hall, E., Brun, Y. V. \& Van Nieuwenhze, M. S. Synthesis of fluorescent Damino acids and their use for probing peptidoglycan synthesis and bacterial growth in situ. Nat Protoc 10, 33-52, doi:10.1038/nprot.2014.197 (2015).

73 Huang da, W., Sherman, B. T. \& Lempicki, R. A. Systematic and integrative analysis of large gene lists using DAVID bioinformatics resources. Nat Protoc 4, 44-57, doi:10.1038/nprot.2008.211 (2009).

74 Kapopoulou, A., Lew, J. M. \& Cole, S. T. The MycoBrowser portal: a comprehensive and manually annotated resource for mycobacterial genomes. Tuberculosis (Edinb) 91, 8-13, doi:10.1016/j.tube.2010.09.006 (2011).

75 Paquette, S. M. L., K.; Longabaugh, W.J.R. BioTapestry now provides a web application and improved drawing and layout tools. F1000Research 5, doi:https://doi.org/10.12688/f1000research.7620.1 (2016).

76 Gupta, S., Stamatoyannopoulos, J. A., Bailey, T. L. \& Noble, W. S. Quantifying similarity between motifs. Genome Bio/ 8, R24, doi:10.1186/gb-2007-8-2-r24 (2007). 
bioRxiv preprint doi: https://doi.org/10.1101/2021.02.25.432019; this version posted February 25, 2021. The copyright holder for this preprint (which was not certified by peer review) is the author/funder, who has granted bioRxiv a license to display the preprint in perpetuity. It is made available under aCC-BY-NC-ND 4.0 International license.

\section{FIGURES}

A

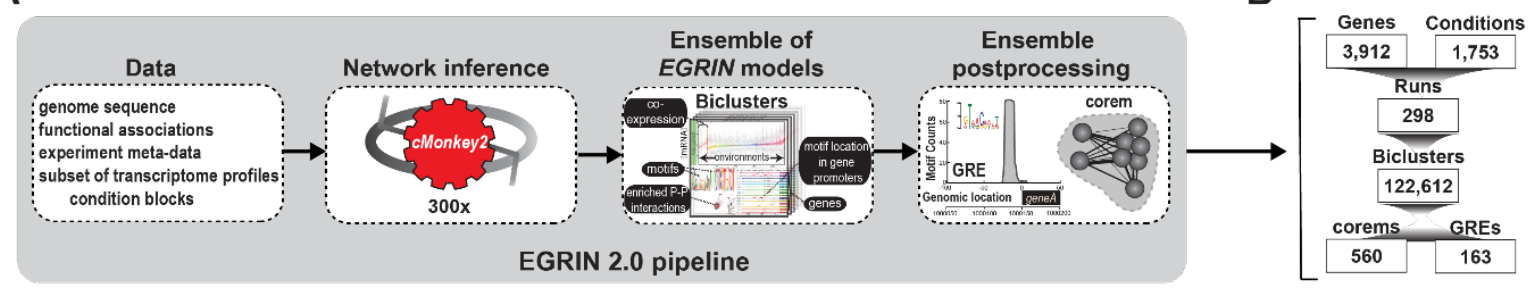

C

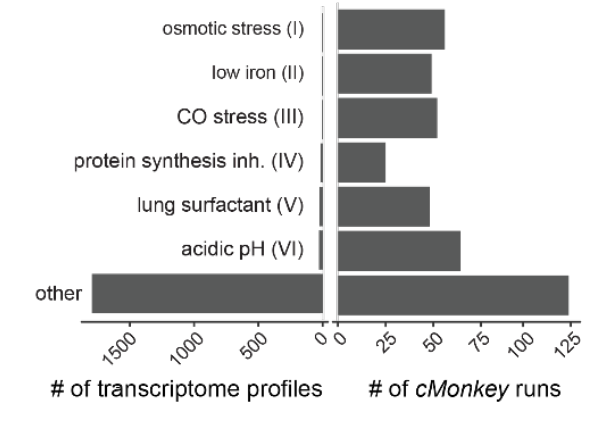

D

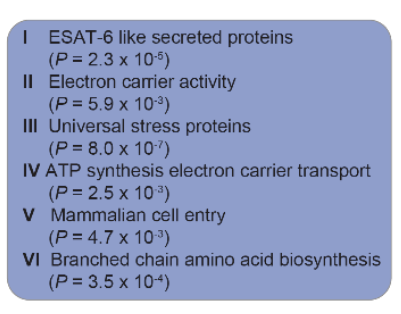

E

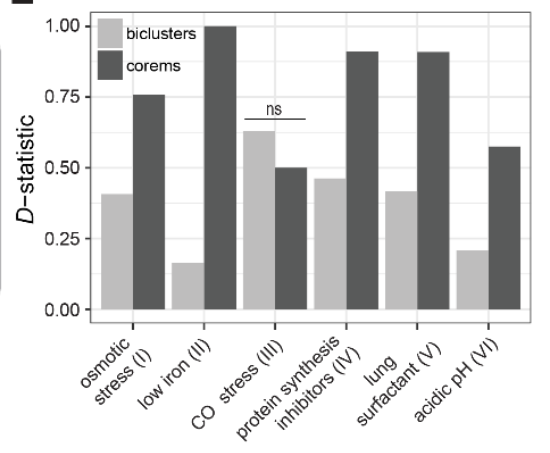

corems

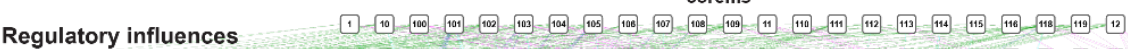

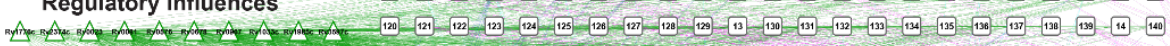

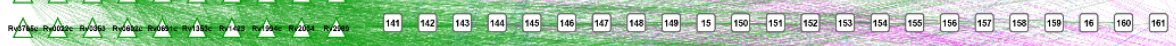

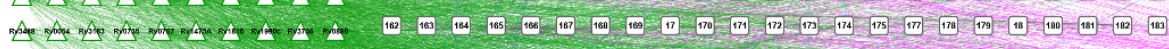

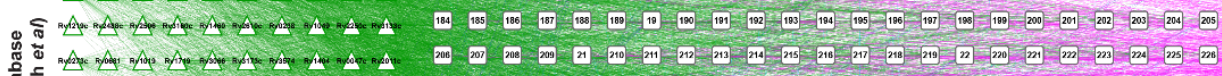

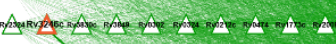

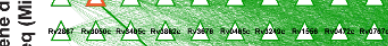

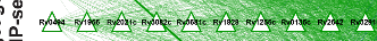
क्ज़

崖

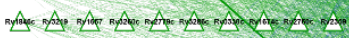

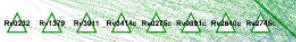

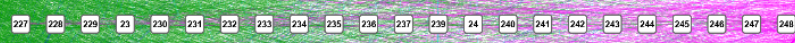

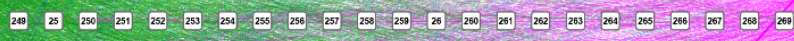

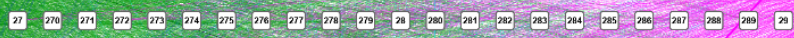

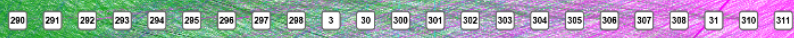

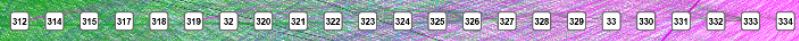

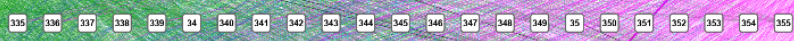

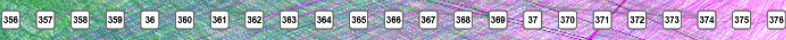

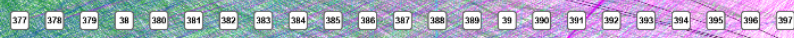

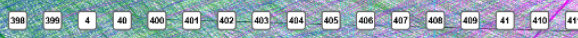

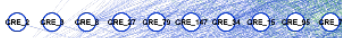

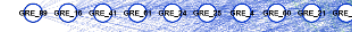

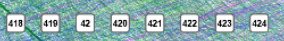

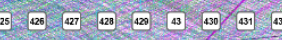

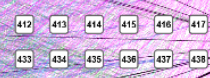

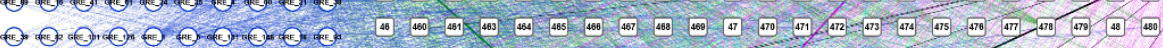

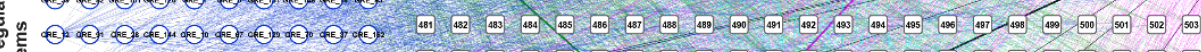

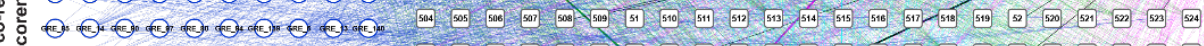

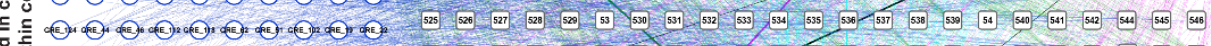

Environmental influences $\begin{gathered}\text { host relevant } \\ \text { stresses }\end{gathered}$
$\begin{gathered}\text { carbon source } \\ \text { availability }\end{gathered}$ $\begin{gathered}\text { antitubercular } \\ \text { treatment }\end{gathered}$ $\begin{array}{ccc}\text { stresses } & \text { avaliability } \\ \text { acidic } y>K & \text { treatment }\end{array}$ b g Y b e b $A$ ch $h$ i $y \mathrm{~g}$ a $i n=d r$ e 10 Genome-wide fitness effects of acidic pH (Tn-seq)

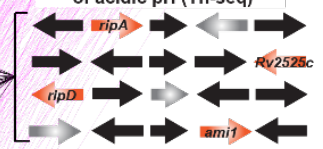

Environments

\begin{tabular}{|c|c|c|}
\hline \multicolumn{3}{|c|}{ in vitro models of host relevant stresses } \\
\hline $\begin{array}{l}\text { a. acidic pH } \\
\text { b. hypoxia } \\
\text { c. DNA damage } \\
\text { d. ROS stress } \\
\text { e. CO stress }\end{array}$ & $\begin{array}{l}\text { f. osmotic stress } \\
\text { g. low oxygen } \\
\text { h. intracellular } \\
\text { i. cell wall stress } \\
\text {. lung surfactant }\end{array}$ & $\begin{array}{l}\text { k. reaeration } \\
\text { I. mutant growth } \\
\text { m. nitrosative stress } \\
\text { n. standard growth } \\
\text { o. low iron }\end{array}$ \\
\hline \multicolumn{3}{|c|}{ in vitro models of host carbon source availability } \\
\hline \multicolumn{3}{|c|}{ 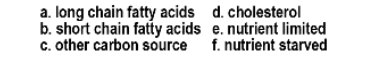 } \\
\hline \multicolumn{3}{|c|}{ in vitro antitubercular treatment } \\
\hline $\begin{array}{l}\text { a. respirat } \\
\text { b. iron sca } \\
\text { c. protein } \\
\text { d. DNA da }\end{array}$ & $\begin{array}{lll}\text { tory inh. } & \text { e. othe } \\
\text { avenger } & \text { f. cellv } \\
\text { inh. } & \text { g. tran } \\
\text { amage } & \text { h. aron }\end{array}$ & $\begin{array}{l}\text { er antibiotics } \\
\text { wall inh. } \\
\text { nscription inh. } \\
\text { matic amides }\end{array}$ \\
\hline
\end{tabular}

Functions enriched in corems PEIPPE unknown devifulence cell wall regulatory conseryed
processes protens hypotheticals

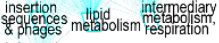
information
pathways cell wall

\& cell
processes 
Figure 1. Overview of EGRIN 2.0 model for M. tuberculosis. (A) The cMonkey algorithm was applied many times to subsets of gene expression data from a large compendia of transcriptome profiles to construct many individual EGRIN models. The cMonkey biclustering algorithm identified sets of genes that have coexpression under a subset of experimental conditions, have a common motif in their promoters and are enriched in protein-protein (P-P) interactions. Individual EGRIN models were integrated into an ensemble and mined to construct the final EGRIN 2.0 model, defining overlapping co-regulated sets of genes (corems) that are statistically associated with specific gene regulatory elements (GREs). (B) A summary of the counts for each feature in the EGRIN 2.0 model for Mtb. (C) Condition blocks representing $<2 \%$ of all transcriptome profiles and their inclusion in cMonkey runs. (D) General theme of most significant functional cluster defined by DAVID ${ }^{73}$ for each condition block shown in (C). (E) Enrichment of functional clusters in (D) for biclusters (EGRIN models containing relevant condition block) and corems of EGRIN 2.0 model, using one-tailed Komogorov-Smirnov (KS) test. We report the KS D-statistic. ns, not significant. (F) The EGRIN 2.0 model of Mtb with 560 corems, each of which is statistically associated with GREs (blue circles) or transcription factors (TFs) from ChIP study by Minch et al ${ }^{17}$ (green triangles). Corems are also statistically associated with environmental influences (pink diamonds) and general mycobacterial functions (teal parallelograms) defined by MycoBrowser ${ }^{74}$. We highlight a signal path through the network that is discussed in detail (bold edges and nodes): genes essential for growth at acidic $\mathrm{pH}$ are significantly enriched in corem 71, which is predicted to be regulated by MtrA and GRE \#11 and implicated in processes related to the cell wall. The inset illustrates ways in which the network model can be used to make actionable predictions. The diagram was generated with Biotapestry ${ }^{75}$. CO: carbon monoxide. inh: inhibitor(s). 


\section{A}

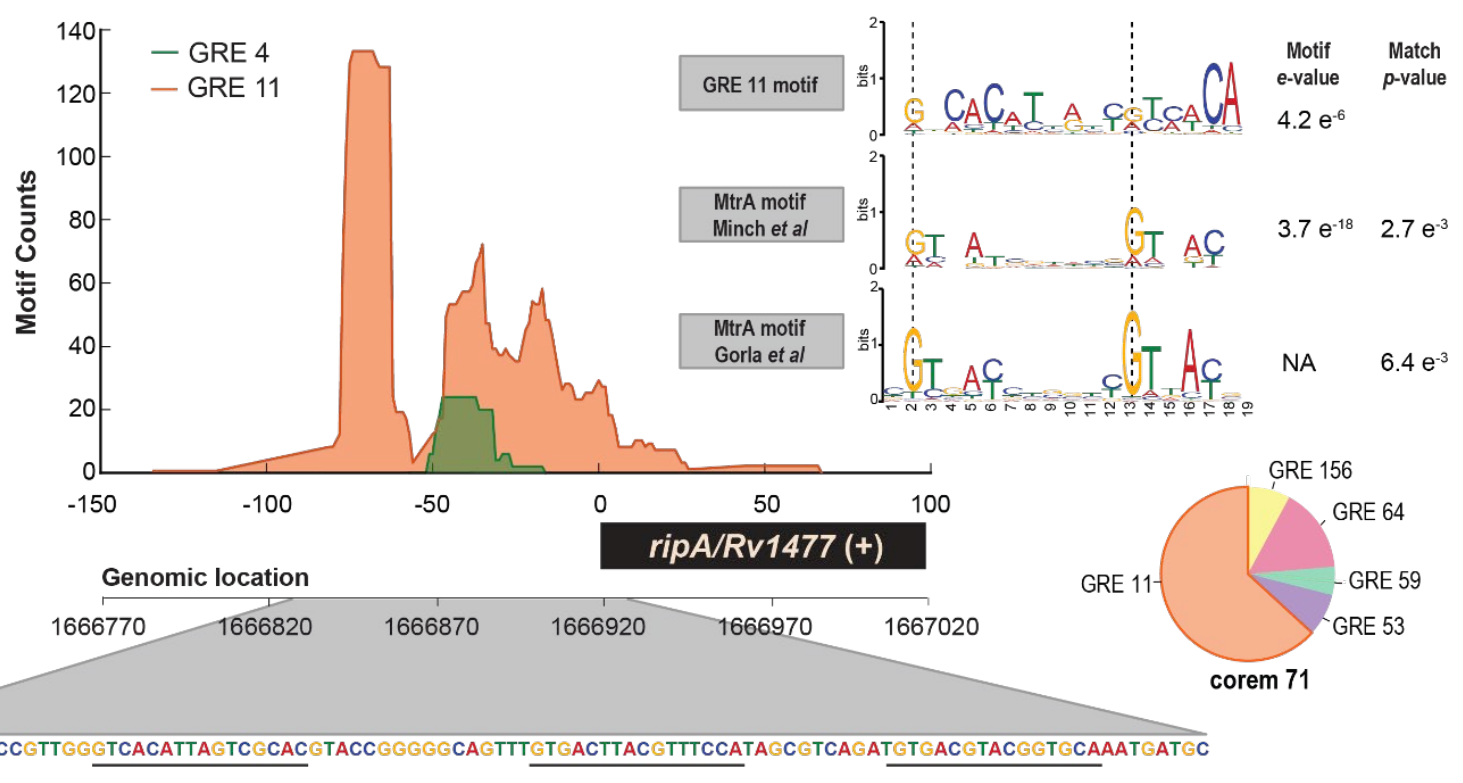

B

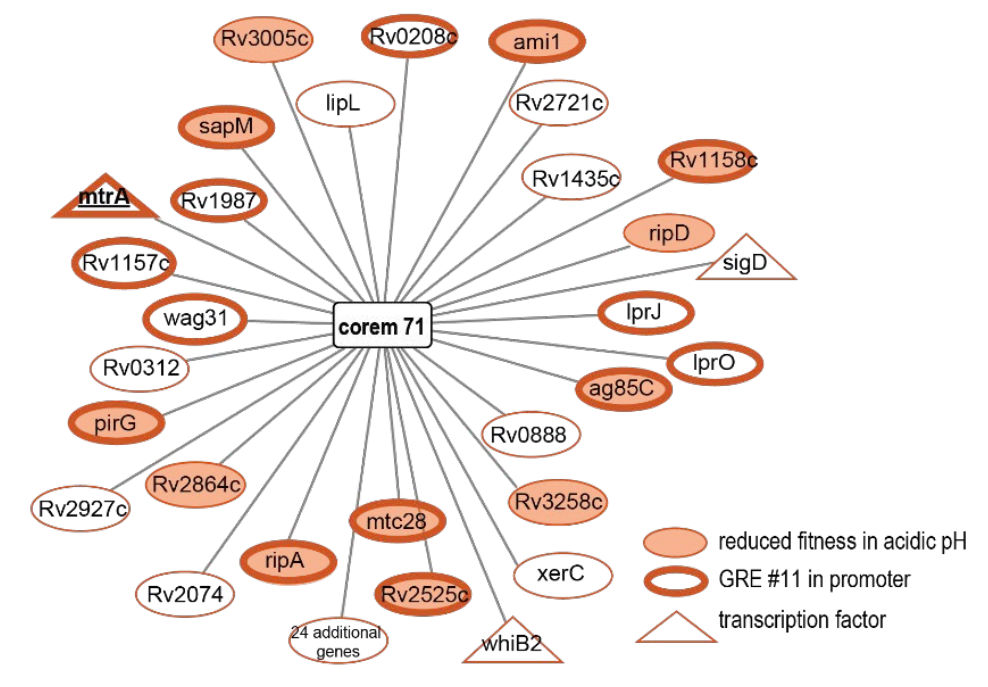

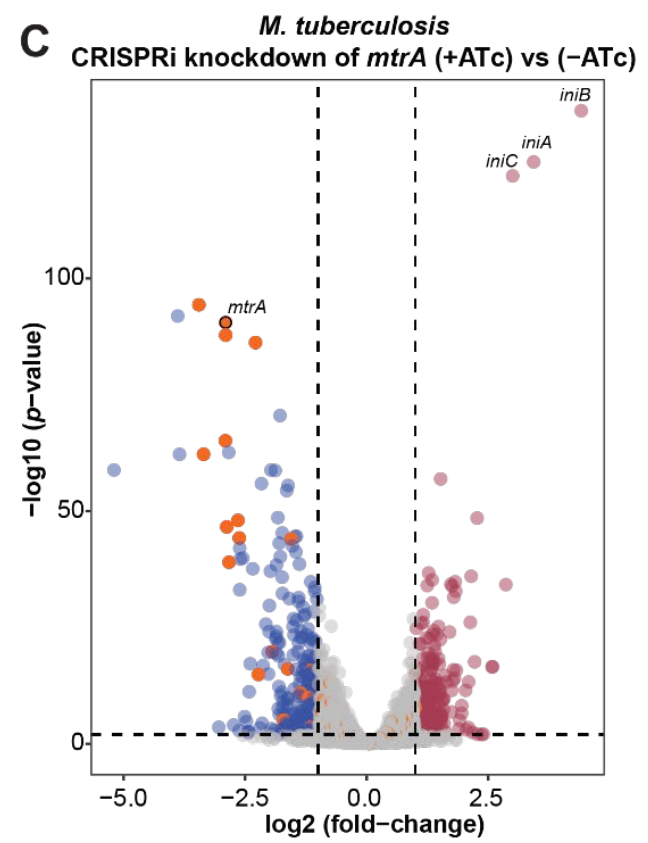

743 Figure 2. Validation of EGRIN 2.0 predicted regulation of corem 71 genes. (A) Promoter architecture of

744 the ripA (Rv1477) promoter predicted by EGRIN 2.0 model. (Top-left) Frequency of GRE alignment to

745 each position in the ripA promoter. GREs are indicated by lines, with GRE \#11 shaded in orange. (Bottom-

746 left) Genome sequence marked with putative GRE \#11 locations. (Top-right) The motif logo of GRE \#11

747 compared with MtrA motifs that were deciphered through analysis of ChIP-seq mapped binding locations.

748 The $e$-values of the motifs and $p$-values from alignment to GRE \# 11 carried out with Tomtom ${ }^{76}$ are shown.

749 NA: not available (Bottom-right) Pie chart represents average predicted influence of GREs on the regulation 
750 of genes in corem 71. (B) Network visualization of genes (ellipses) in corem 71. (C) Volcano plot of 751 differentially expressed genes for induced and uninduced CRISPRi knockdown of $m t r A$ with sgRNA2 in 752 Mtb. The significantly differentially expressed genes were selected by $p$-value $<0.01$ and absolute $\log 2$

753 fold-change $>1$. Dots represent different genes, with labels for particular genes of interest. Grey dots are 754 genes without significant different expression, red dots are significantly up-regulated genes ( $N=266$ genes) 755 and blue dots are significantly down-regulated genes $(N=240$ genes). The orange dots are all genes of 756 corem 71. 

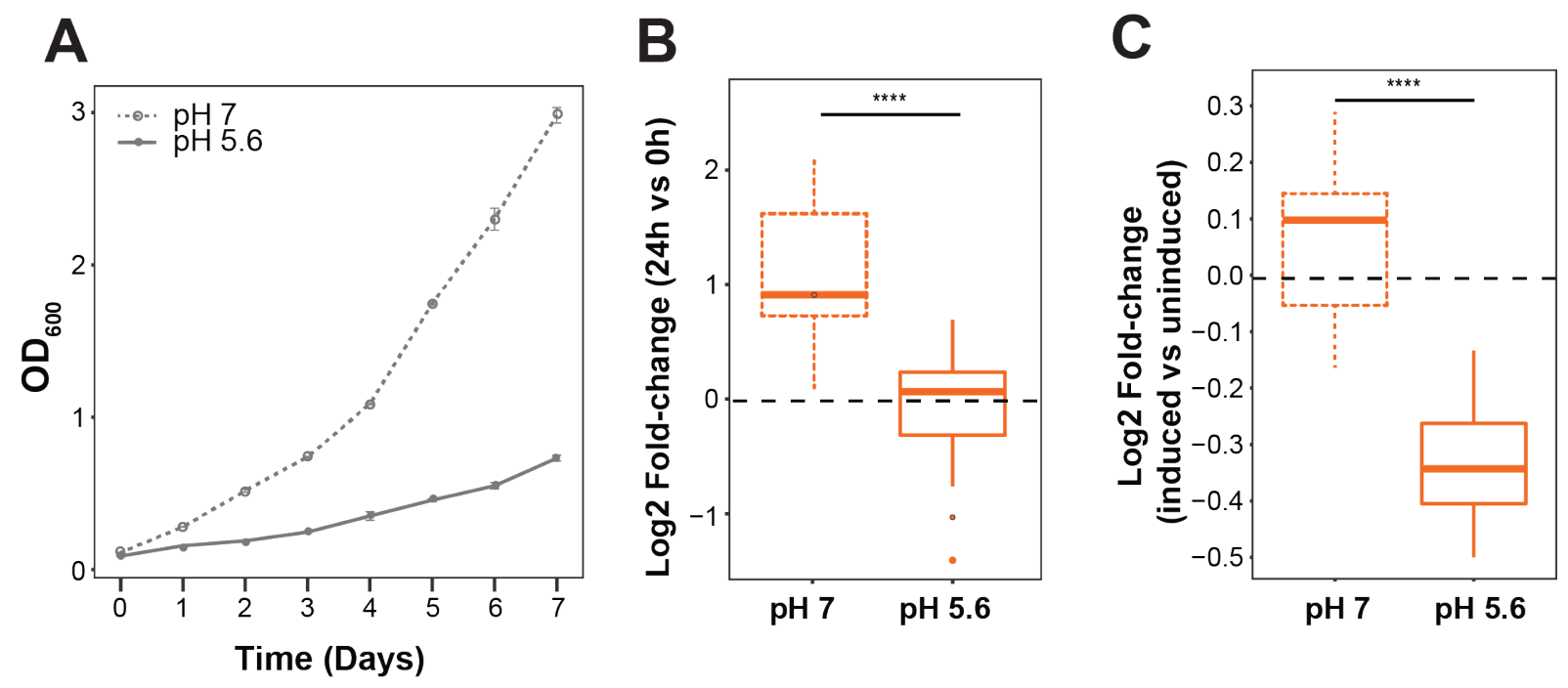

Figure 3. Characterization of corem 71 in acidic pH. (A) Growth of wild-type Mtb H37Rv in 7H9-rich media buffered to either $\mathrm{pH} 7$ (circles, dashed line) or pH 5.6 (squares, solid line). Growth was monitored daily by optical density at $650 \mathrm{~nm}$. Points are the average of three biological replicates and error bars represent standard deviation. (B) Boxplot representing log2 fold-change of corem 71 genes with reduced fitness in acidic $\mathrm{pH}$ for wild-type Mtb H37Rv in 7H9-rich media buffered to either $\mathrm{pH} 7$ (dotted line) or pH 5.6 (solid line). Data are for three biological samples comparing expression at $24 \mathrm{~h}$ vs $0 \mathrm{~h}$. (C) Boxplot representing $\log 2$ fold-change of Mtb H37Rv with inducible overexpression of $m t r A$ for corem 71 genes with reduced fitness. Data are for three biological samples in 7H9-rich media buffered to neutral $\mathrm{pH} 7$ (dotted line) or acidic pH 5.6 (solid line) comparing induced (+ATc) vs uninduced (-ATc) overexpression of $m$ trA. Statistical significance was calculated as $p$-value with Student's T-test. ****: $p$-value $<0.0001$. 
A

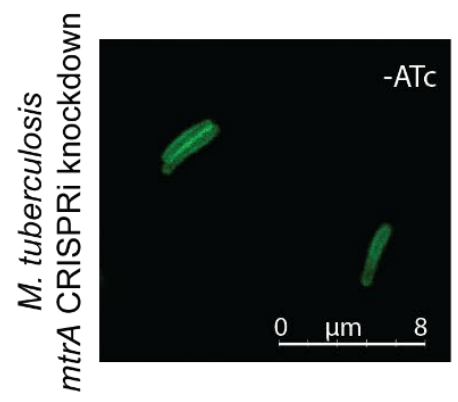

B

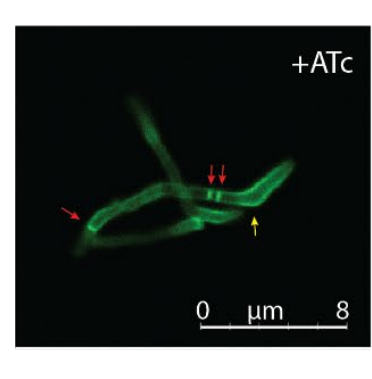

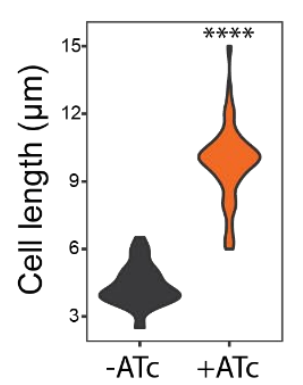

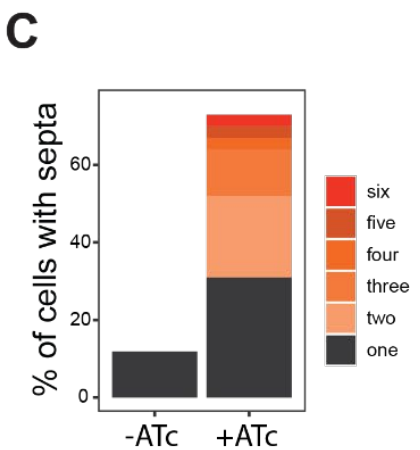

772 Figure 4. MtrA controls cell division in M. tuberculosis. (A) Example micrographs of uninduced (-ATc) 773 and induced (+ATc) CRISPRi knockdown of $m$ trA with sgRNA5 in Mtb. After knockdown, cells were

774 labeled with HCC-amino-D-alanine (HADA) for $20 \mathrm{~h}$. Red arrows indicate multiple septa and yellow arrow

775 indicates the curved shape phenotype. (B) Violin plots showing the cell lengths of uninduced and induced

776 CRIPSRi knockdown of mtrA with sgRNA5 in Mtb. Significance was determined by Student's T-test. ****:

$777 p$-value $<0.0001$. (C) Bar plots representing the percentage of cells that contain 1 or more septa for 778 uninduced and induced CRIPSRi $m t r A$ knockdown with sgRNA5 in Mtb. The colored sections within the 779 bars represent the proportions of cells with different numbers of septa. Representative results from two 780 independent experiments are shown. 

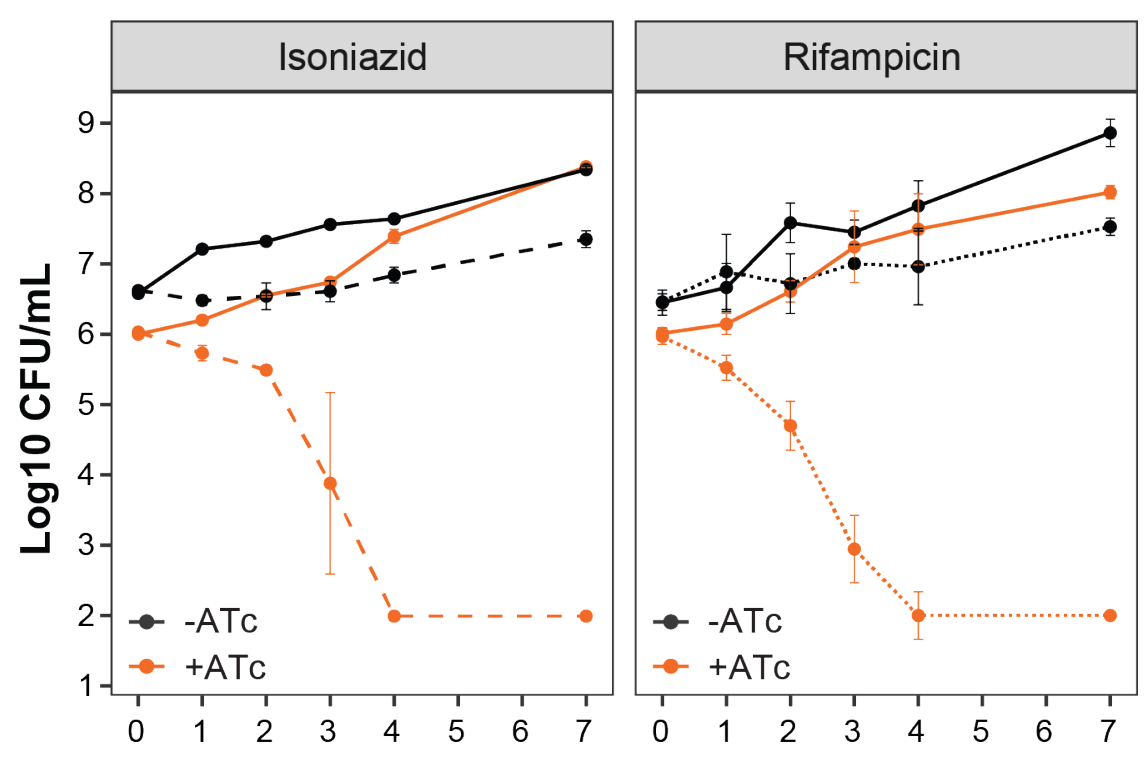

\section{Days}

784 Figure 5. The knockdown of $m$ tr $A$ sensitizes $M$. tuberculosis to frontline TB drugs. Colony forming units 785 (CFU) over a period of 7 days for untreated (solid lines) and $0.18 \mu \mathrm{g} / \mathrm{mL}$ isoniazid or $0.006 \mu \mathrm{g} / \mathrm{mL}$ 786 rifampicin treated (dotted lines) liquid cultures of uninduced (black) and induced (orange) CRIPSRi 787 knockdown of mtrA with sgRNA5 in Mtb. Error bars show the standard deviation from three biological 788 samples. Representative results from two experiments are presented. 
bioRxiv preprint doi: https://doi.org/10.1101/2021.02.25.432019; this version posted February 25, 2021. The copyright holder for this preprint (which was not certified by peer review) is the author/funder, who has granted bioRxiv a license to display the preprint in perpetuity. It is made available under aCC-BY-NC-ND 4.0 International license.

791 Tables

792 Table 1. Corem 71 genes with predicted regulation by MtrA. na: not applicable; ns: not significant

\begin{tabular}{|c|c|c|c|c|c|c|c|}
\hline Gene & Name & Description & $\begin{array}{l}\text { Gene with } \\
\text { reduced } \\
\text { fitness at } \\
\text { acidic pH }\end{array}$ & $\begin{array}{l}\text { Gene with } \\
\text { GRE \#11 in } \\
\text { promoter }\end{array}$ & $\begin{array}{c}\text { Mtb } m \text { trA } \\
\text { knockdown } \\
\text { +ATc vs -ATc } \\
\text { Log2 FC } \\
(p<0.01)\end{array}$ & $\begin{array}{c}\text { Msm } \\
\text { homolog }\end{array}$ & $\begin{array}{c}\text { Msm mtrA } \\
\text { knockdown } \\
\text { +ATc vs -ATc } \\
\text { Log2 FC } \\
(p<0.01) \\
\end{array}$ \\
\hline Rv0040c & $m t c 28$ & secreted protein (core mycobacterial gene) & & $\mathrm{x}$ & -2.90 & MSMEG_6919 & -4.13 \\
\hline Rv0129c & $a g 85 C / f b p C$ & cell wall trehalose dimycolate biosynthesis & $x$ & $x$ & -1.15 & MSMEG_3580 & -3.77 \\
\hline$R v 1157 c$ & & conserved protein (core mycobacterial gene) & & $\mathrm{x}$ & -1.36 & MSMEG_5153 & -4.45 \\
\hline$R v 1477$ & ripA & peptidoglycan endopeptidase & $x$ & $x$ & -2.29 & MSMEG_3145 & -0.80 \\
\hline Rv1566c & ripD & peptidoglycan endopeptidase & $x$ & $\mathrm{x}$ & -2.65 & MSMEG_3477 & -3.1 \\
\hline$R \vee 1987$ & & probable chitinase & & $x$ & -3.36 & & \\
\hline Rv2145c & $\operatorname{wag} 31$ & essential gene, cell division protein DivIVA & na & $\mathrm{x}$ & -0.88 & MSMEG_4217 & ns \\
\hline$R v 2525 c$ & & peptidoglycan glycoside hydrolase (core mycobacterial gene) & $\mathrm{x}$ & $\mathrm{x}$ & -0.72 & MSMEG_6815 & ns \\
\hline$R \vee 3310$ & sapM & acid phosphatase & $x$ & & -0.84 & & \\
\hline
\end{tabular}

